Check for updates

Cite this: Mater. Chem. Front., 2020, 4, 155

Received 11th September 2019 Accepted 1st October 2019

DOI: 10.1039/c9qm00571d

rsc.li/frontiers-materials

\section{Self-assembly of chiral supra-amphiphiles}

\author{
Guanghui Ouyang ${ }^{a}$ and Minghua Liu (D) *ab
}

\begin{abstract}
Supra-amphiphiles provide a brand-new powerful strategy for the construction of functional materials and have shown fascinating prospects in a variety of fields. When merged with chirality science, the concept of chiral supra-amphiphiles arises, which is an important subclass of supra-amphiphiles. In this review, we have provided a general introduction to the concept and design principle of chiral supraamphiphiles as well as their self-assemblies. We have also highlighted some progress of chiral supraamphiphiles in several application fields, such as chiroptical switches, chiral recognition, chiral catalysis and chiral luminescent soft materials. Although some elegant reviews have comprehensively looked back at the development and achievement of supra-amphiphiles, their research progress in chirality science has not been well summarized. We hope this review will provide useful guidance and understanding of self-assembly of chiral supra-amphiphiles, which we believe will benefit scientists in both colloidal chemistry and chirality science.
\end{abstract}

\section{Introduction}

Amphiphiles are one type of unique chemical compounds in colloidal science, which usually contain two main parts, a hydrophilic head and a lipophilic tail. ${ }^{1-11}$ They show excellent

${ }^{a}$ Beijing National Laboratory for Molecular Science, CAS Key Laboratory of Colloid, Interface and Chemical Thermodynamics, Institute of Chemistry, Chinese Academy of Sciences, ZhongGuanCun, North First Street 2, 100190, Beijing, China.

E-mail: liumh@iccas.ac.cn

${ }^{b}$ University of Chinese Academy of Sciences, Beijing 100049, P. R. China self-assembly capability in aqueous solution to give varied structures like vesicles, micelles and tubules, ${ }^{12-18}$ which not only provide satisfactory platforms for the mimicking of lipid bilayers in living organisms, ${ }^{19-21}$ but also exhibit extraordinary applications in modern industry and humans' daily lives, such as the preparation of crude oil viscosity reducers ${ }^{22}$ and the production of soaps and detergents. ${ }^{23-26}$ On the other hand, the fast development of supramolecular chemistry, which is based on a variety of non-covalent interactions, ${ }^{27-31}$ brings new opportunities for organic chemistry, materials chemistry and colloidal chemistry. ${ }^{32-36}$

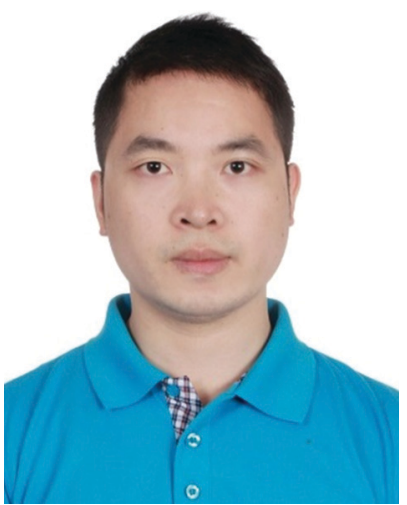

Guanghui Ouyang
Guanghui Ouyang was born in Hunan province, China in 1987. He received his $B S c$ from the University of Science and Technology of China (USTC) in 2010 and PhD degree from the Institute of Chemistry, Chinese Academy of Sciences (ICCAS) in 2015. He is currently an associate professor at ICCAS. His research interests focus on the design and application of chiral supramolecular $\pi$-gels and catalysts.

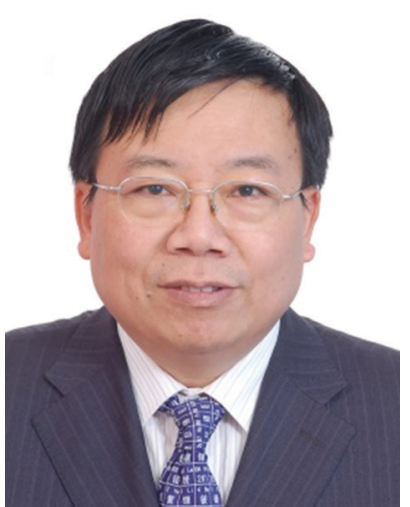

Minghua Liu
Minghua Liu graduated from Nanjing University in 1986. He received his $P h D$ from Saitama University, Japan in 1994. After doing postdoctoral research at the Institute of Physical and Chemical Research (RIKEN) and at the Tokyo University of Agriculture and Industry, he joined the Institute of Photographic Chemistry in 1998 and then the Institute of Chemistry, CAS in 1999 and has been working as a professor till now. His research interests cover supramolecular chemistry, colloid and interface science, self-assembly and gel-based soft nanomaterials, in particular, chirality issues in supramolecular and self-assembly systems. 
Supra-amphiphiles, a new bridge between colloidal chemistry and supramolecular chemistry, were born and rapidly gained increasing attention with the pioneering work of Zhang and other scientists around the world. ${ }^{37-41}$ Generally, in a narrow sense, supra-amphiphiles represent amphiphiles in which the hydrophobic part and the hydrophilic part are connected via noncovalent interactions or dynamic covalent bonds. ${ }^{42-45}$ But in a larger sense, a two-component or multi-component supramolecular system constructed by noncovalent interactions or dynamic covalent bonds, which shows an amphiphilic property, can also be regarded as a supra-amphiphile. ${ }^{46-57}$ Several important reviews have well summarized the recent progress in supra-amphiphiles. ${ }^{58-61}$ For example, Zhang and co-workers have systematically outlined the definition, categorization, characterization and applications of supra-amphiphiles in their leading reviews. ${ }^{62,63}$ Hao and co-workers also nicely overviewed the preparation of vesicles from supramolecular amphiphiles using varied strategies. ${ }^{61,64}$

Meanwhile, chirality and chiral materials have been gaining increasing attention from the chemical community in the past decades due to their wide and attractive applications in chiral separation, chiral catalysts, chiral luminescent materials, medicine and sensors. ${ }^{65-73}$ Chiral self-assembly of amphiphiles is one of the sophisticated ways to fabricate chiral structures and materials, ${ }^{74-80}$ and also plays a critical role in the understanding of many chiral phenomena in life systems, considering that some membranes and organelles constructed from lipid molecules are actually chiral structures. ${ }^{81,82}$ When merging with chiral characteristics, a new application field for the concept of supra-amphiphiles arises, that is chiral self-assembly of supraamphiphiles. It provides a powerful alternative strategy for the preparation of chiral functional materials and has shown unique advantages in a variety of chirality fields owing to its dynamic nature based on non-covalent interactions. However, in contrast to the furious research progress and reviews of self-assembly of chiral amphiphiles, ${ }^{83,84}$ the summary or overview of selfassembly of chiral supra-amphiphiles has not been timely processed. In this review, we will introduce some recent progress in this subfield of supra-amphiphiles, mainly focusing on the self-assembly of chiral supra-amphiphiles initiated by typical non-covalent interactions, such as hydrogen bonds, coordination bonds and electrostatic interactions. Besides, cyclodextrin is a famous chiral host and can form many host-guest complexes. Thus, the self-assembly of chiral supra-amphiphiles derived from cyclodextrin has also been summarized. We hope that this review will contribute to the development of supra-amphiphiles in chirality science (Fig. 1).

\section{Self-assembly of chiral supra-amphiphiles}

\subsection{Self-assembly of hydrogen bond based chiral supra-amphiphiles}

Hydrogen bonds are widely used in supramolecular chemistry and colloidal chemistry because of their well-oriented

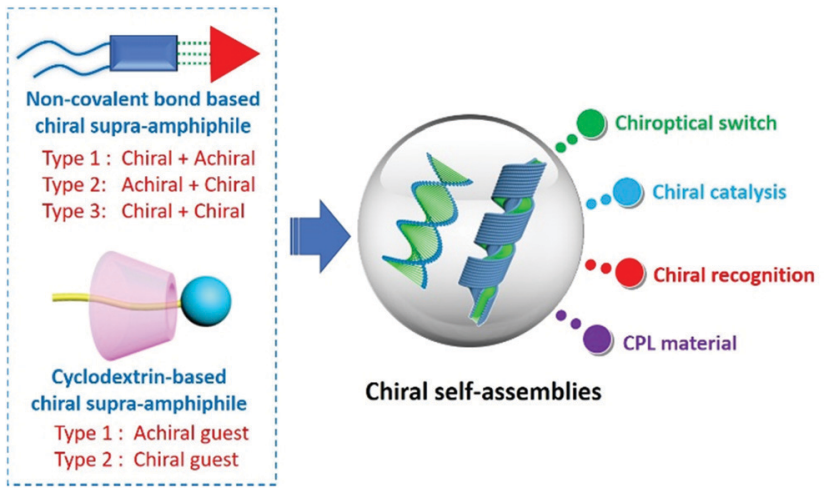

Fig. 1 Illustration of the design strategy of chiral supra-amphiphiles and their chiral self-assemblies applied to several chiral fields. Notes: the types (type 1, type 2 and type 3 ) in the upper left corner represent the chiral status of each part of the supra-amphiphile.

directionality and adequate bond energy as well as reversibility. ${ }^{85-87}$ Many elegant supra-amphiphiles derived from single, double, and triple hydrogen-bonds and even quadruple hydrogenbonds had been well established. ${ }^{88-90}$ Their self-assembly mechanism and applications have also been investigated. However, the design and self-assembly of hydrogen-bond based chiral supra-amphiphiles are relatively less studied, partly due to the complexity of these chiral systems and the challenge of supramolecular chirality formation and transfer. Some representative examples of hydrogen-bonded chiral supraamphiphiles and their self-assembly will be carefully discussed in the following content.

Liu and co-workers reported a chaperone gelator LHC18, which is composed of a long alkyl chain, an L-histidine and a urea connecting bond. The imidazole unit on this gelator could form electrostatic interaction assisted hydrogen bonds with varied carboxylic acid compounds, as shown in Fig. 2, efficiently yielding supra-amphiphiles and two-component supramolecular gels in water/organic co-solvents.

Through this strategy, they prepared several kinds of supraamphiphiles and investigated their chiral self-assembly and chiral functions. For example, they found that this chaperone

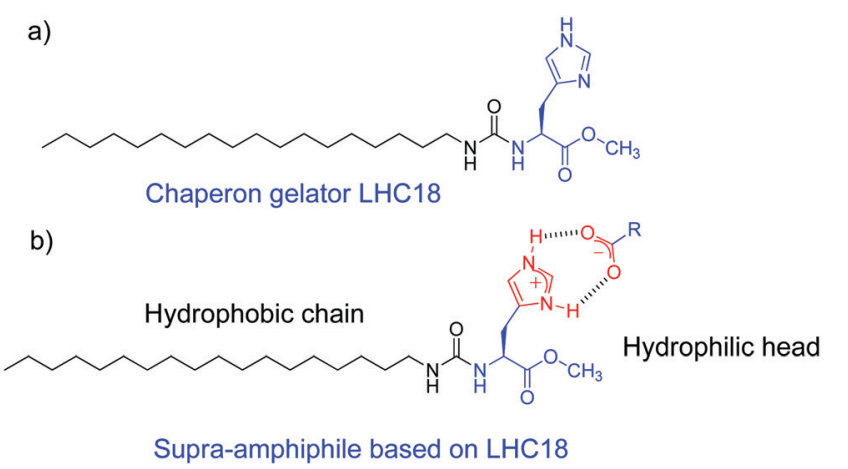

Fig. 2 The molecular structures of chaperone gelator LHC18 and supraamphiphiles based on LHC18. Electrostatic interaction assisted hydrogen bonds between imidazole and carboxylic acid lead to the formation of the hydrophilic head. 
gelator gelled all the non-gelling proteinogenic L-amino acids and their counterpart D-amino acids in $\mathrm{DMF} / \mathrm{H}_{2} \mathrm{O}$ co-solvents. Varied nanostructures such as nanospheres, nanoplates, nanofibers, nanotwists, nanotubes and mircoclusters could be observed via SEM, depending on the type of amino acids. ${ }^{91}$

They further selected several azobenzene carboxylic acid compounds to form supra-amphiphiles with the chaperone gelator. As shown in Fig. 3, three kinds of azobenzene compounds were used, AZO-1 contains two alkoxyl spaced carboxylic acid pendants, and AZO-2 contains one alkoxyl spaced carboxylic acid pendant, while AZO-3 contains two directly linked carboxylic acid groups. All the three AZO compounds could not form gels individually, however, when they were co-assembled with the chaperone gelator LHC18, two-component supramolecular gels were efficiently prepared via the formation of supra-amphiphiles. SEM images showed that loose nanotubes, nanotubes and nanosheets were obtained depending the type of AZO compounds. Upon the gelling process, the molecular chirality localized at histidine could be transferred to azobenzene assemblies, showing CD signals in the absorption region of the azobenzene rings. However, the photo-triggered cis-trans transformation of the azobenzene unit in these supra-amphiphile assemblies showed obvious differences. As a result, the optical and chiroptical properties were totally different. The LHC18/AZO-1 and LHC18/AZO-3 supra-amphiphiles showed little change in the CD spectra, while the LHC18/AZO-2 supra-amphiphile showed considerable reduction in the CD intensity. Furthermore, alternate UV and visible light irradiation could realize reversible gel-sol transformation for the LHC18/AZO-2 supra-amphiphile based gel. And the CD signals in these two states could also be repeated for at least five cycles. The authors suggested that a flexible spacer such as alkoxy on the azobenzene carboxylic acid contributes to reversible transformation of these properties. Combining the photo-responsive properties of achiral azobenzene and the chiral properties of the gelator, a chiroptical switch was newly constructed, which well reflected the unique feature of non-covalent bonded supra-amphiphiles. ${ }^{92}$

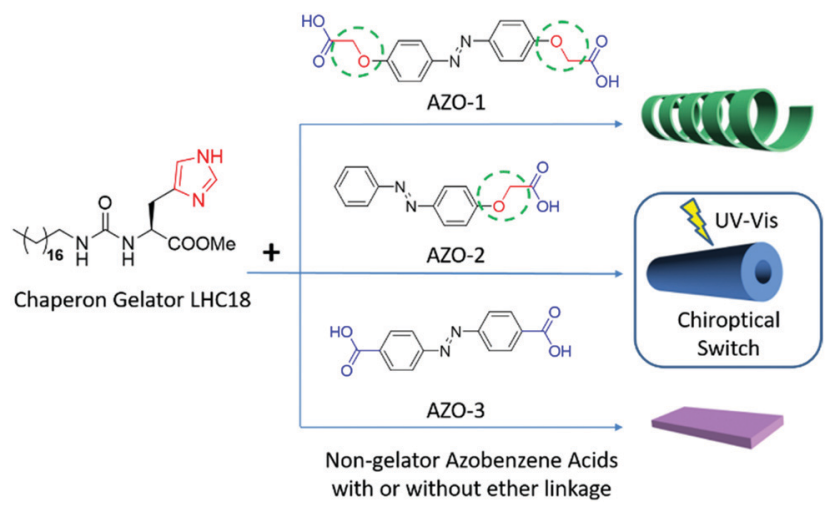

Fig. 3 The molecular structures of the chaperone gelator and three kinds of azobenzene carboxylic acids. The right figures are cartoon images of the nanostructures of the corresponding supra-amphiphile gels. (Reprinted with permission from ref. 92. Copyright (C) 2017 American Chemical Society.)
In another case, Liu and co-workers prepared a tetra-pendant supra-amphiphile from the chaperone gelator (enantiomers LHC18 or DHC18) and a porphyrin with four carboxylic acid groups (TCCP). As shown in Fig. 4, they found that the LHC18/ TCCP supra-amphiphile could form right-handed hierarchical microflowers, while the DHC18/TCCP supra-amphiphile gave left-handed ones. This meant that the supramolecular chirality of supra-amphiphile self-assemblies followed the molecular chirality of the histidine moiety. Moreover, these chiral nano- or microstructures showed macroscopic enantioselective recognition towards amino acids enantiomers via contact angle measurements. ${ }^{93}$

This special strategy of hydrogen-bonded supra-amphiphiles could also be applied to catalysis. In 2018, Liu and his colleagues reported a new kind of supra-amphiphile based on natural product hemin and chaperone gelator $\mathrm{L}^{-}$or $\mathrm{D}-\mathrm{HC} 18 .{ }^{94}$ As shown in Fig. 5, hemin/LHC18 and hemin/DHC18 supra-amphiphiles

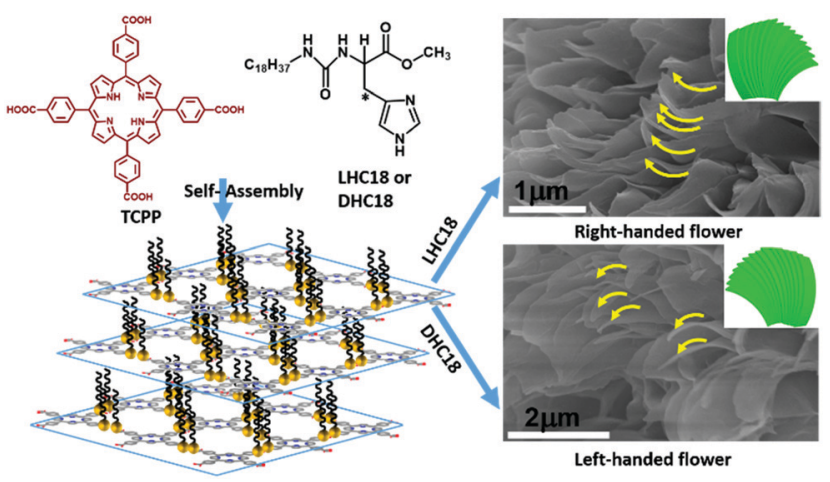

Fig. 4 The molecular structures of the chaperone gelator and porphyrin analogues with four carboxylic acid groups. And the illustration of formation and self-assembly of supra-amphiphiles. (Reprinted with permission from ref. 93. Copyright (C) 2017 American Chemical Society.)
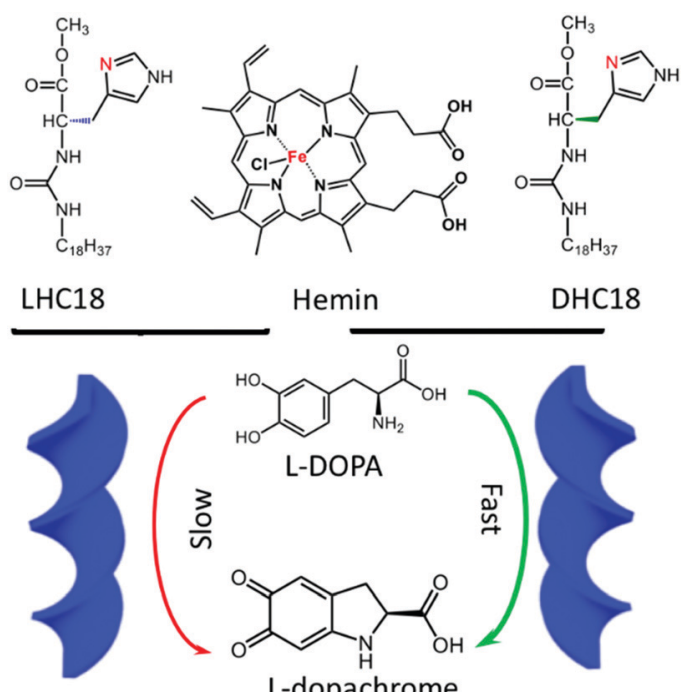

Hemin

DHC18

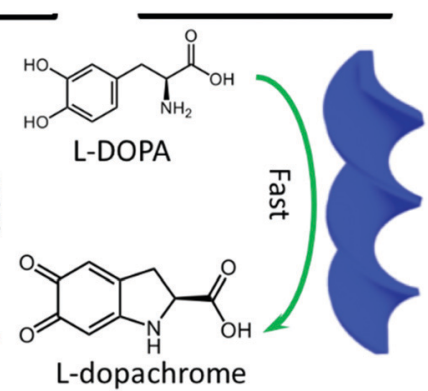

Fig. 5 The molecular structures of supra-amphiphiles based on hemin and the chaperone gelator. hemin/DHC18 supra-amphiphile gels showed a faster catalytic oxidation rate for L-DOPA. (Reprinted with permission from ref. 94. Copyright (C) 2018 John Wiley \& Sons, Inc.) 
can self-assemble into right-handed and left-handed helical ribbons respectively, which were clearly observed from SEM images and supported by the CD spectra. Although natural hemin is achiral, it showed supramolecular chirality when participating in the network of supra-amphiphiles via efficient chirality transfer. The authors applied these supra-amphiphile chiral nanostructures to the catalysis of 3,4-dihydroxyl-L-phenylalanine (L-DOPA). L-DOPA can be oxidized by hemin, giving L-dopachrome, which is also a chiral compound. It was found that the hemin/DHC18 supra-amphiphile showed a much faster catalytic rate compared with the counterpart hemin/LHC18. They also proposed a possible mechanism for this rate selectivity. First, hemin could capture the chiral sense of LHC18 or DHC18, which was confirmed by CD spectra. Second, when the chiral L-DOPA targets the chiral catalytic center of hemin, chiral matching might determine the catalytic rate-limiting step. As a result, favourable chiral matching between L-DOPA and hemin/DHC18 amphiphile assemblies led to a higher catalytic rate. This work provided an interesting case of integration of supramolecular catalysis and supra-amphiphiles.

Hydrogen bonds between carboxylic acid and amines, such as alkyl amines, aromatic amines and nitrogen-containing heterocyclic compounds, are widely utilized for the design and preparation of supramolecular architectures. Liu and co-workers designed a series of L-glutamic acid amphiphiles containing varied long alkyl chains, which are abbreviated as $\mathrm{L}_{-} \mathrm{C}_{n} \mathrm{GAc}$ ( $n$ represents the number of carbon atoms on the long alkyl chain). As shown in Fig. 6, two $\mathrm{L}_{-} \mathrm{C}_{n} \mathrm{GAc}$ molecules and two 4,4'-bipyridine molecules could form supra-amphiphiles based on four hydrogen bonds between carboxylic acid and pyridine. ${ }^{95}$ This supra-amphiphile successfully self-assembled into nanoplate structures. Most interesting, adding more 4,4'-bipyridine lead to the formation of a sandwich supra-amphiphile, in which the extra 4,4'-bipyridine molecules are inserted into the original two 4,4'-bipyridine. Under this condition, chiral nanotwists were obtained. Through changing the ratio between the two components, the structure of the supra-amphiphile could be

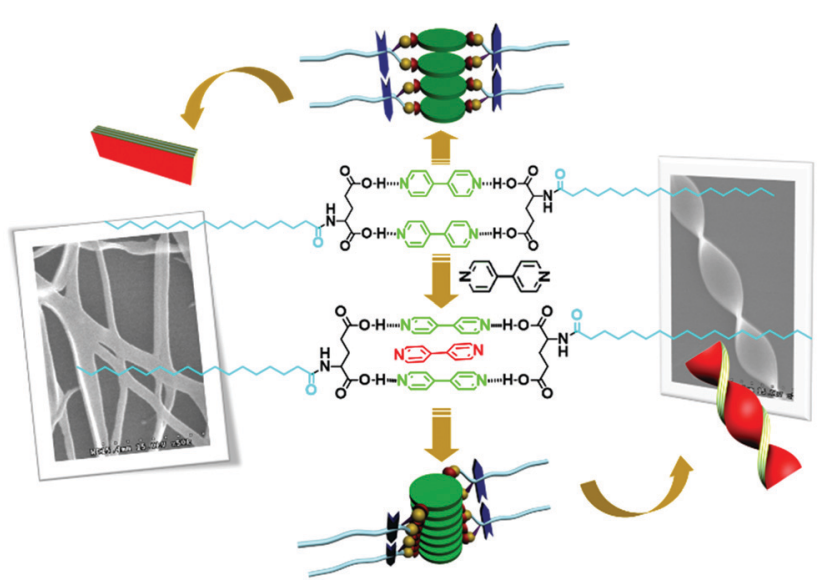

Fig. 6 The molecular structures of supra-amphiphiles based on different mole ratios of $\mathrm{L}_{-} \mathrm{C}_{n} \mathrm{GAC}$ and $4,4^{\prime}$-bipyridine and illustration of selfassembly. (Reprinted with permission from ref. 95. Copyright (C) 2011 John Wiley \& Sons, Inc.) effectively tuned, leading to distinct self-assembly modes, nanostructures and chiroptical properties.

Jiao and co-workers reported a quadruple hydrogen-bonded chiral supra-amphiphile from chiral amphiphilic glutamate compound $\mathrm{L}-\mathrm{GDE}$ and achiral porphyrin derivative TCPP. ${ }^{96}$ This chiral supra-amphiphile could form various hierarchical composite film nanostructures in mixed solvents such as DMF/ $\mathrm{H}_{2} \mathrm{O}$ and $\mathrm{DMF} / \mathrm{CHCl}_{3}$. As shown in Fig. 7, the supra-amphiphile in $\mathrm{DMF} / \mathrm{H}_{2} \mathrm{O}(\mathrm{v} / \mathrm{v}=3 / 7)$ self-assembled into brick-like structures, while in $\mathrm{DMF} / \mathrm{CHCl}_{3}(\mathrm{v} / \mathrm{v}=2 / 8)$ flower-like structures are observed. They found that the intermolecular hydrogen-bonds between different solvents could change the molecular packing mode, which significantly altered the self-assembled nanostructures and triggered supramolecular chirality inversion as supported by circular dichroism signals. These two films showed different chiral recognition ability towards amino acid solutions via the measurement of contact angles.

Multiple hydrogen bonds are also utilized to synthesize chiral supra-amphiphiles. Zhao and co-workers reported two $\mathrm{N}$-terminated aromatic amino acid derivatives, named NSer and PSer. They formed achiral $2 \mathrm{D}$ thin plates in $\mathrm{DMSO} / \mathrm{H}_{2} \mathrm{O}$ via antisolvent methods. They successfully obtained a single crystal of PSer.

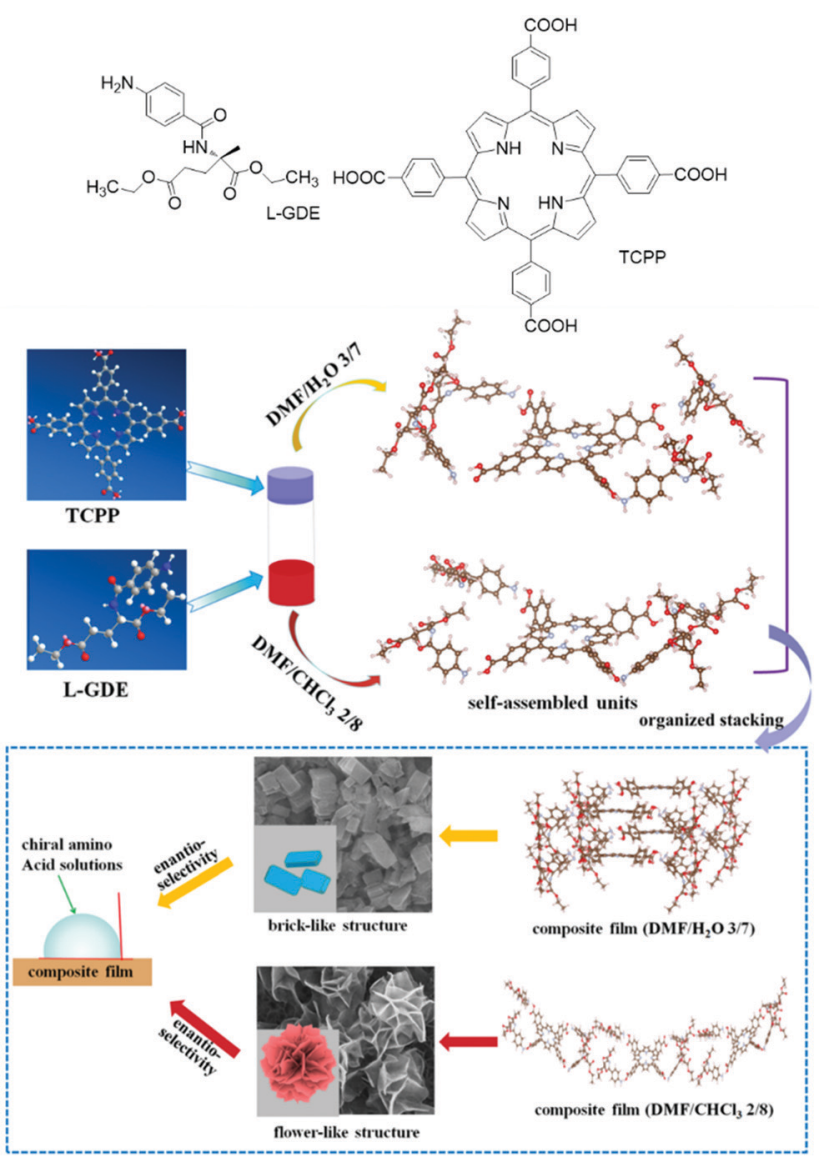

Fig. 7 The chemical structure of TCPP and L-GDE and schematic illustration of different self-assembly pathways under different co-solvents as well as their nanostructures and chiral recognition via contact angle tests. (Reprinted with permission from ref. 96. Copyright (C) 2019 American Chemical Society.) 
There are $\pi-\pi$ stacking (3.48 $\AA$ ) interactions between pyrene units and two $\mathrm{CH}-\pi$ interactions ( $2.86 \AA$ and $2.68 \AA$ ), which contribute to a double-layered morphology. Combined with XRD data, they pointed out that a well-defined lamellar structure was formed with a 26 A-thick double-layer. When co-assembled with achiral melamine derivative ${ }^{\mathrm{Bn}} \mathrm{Mm}$, oppositely chiral nanotubes were obtained for P-L-Ser $/{ }^{\mathrm{Bn}} \mathrm{Mm}$ and P-D-Ser $/{ }^{\mathrm{Bn}} \mathrm{Mm}$, which was further supported by mirror-image CD signals. Fourier transform infrared spectroscopy (FT-IR) indicated that duplex hydrogen bonds between the melamine moiety and carboxylic acid existed in the co-assembly system, which contributes to the formation of a C3-like supra-amphiphile. The supra-amphiphile adopted an asymmetric arrangement, significantly improved the anisotropy factor value and successfully afforded chiral nanostructures, such as twisted ribbons, helical fibers and helical nanotubes. This work provides a new example of the design and selfassembly of chiral supra-amphiphiles via multiple hydrogen bonds (Fig. 8 and 9). ${ }^{97}$

Das and co-workers synthesized a cationic achiral $\mathrm{C}_{3}$-symmetric gelator $\mathrm{L}^{-} \mathrm{Cl}^{-}$. It could form a light yellow opaque gel via a heatingcooling procedure in $\mathrm{MeOH} / \mathrm{H}_{2} \mathrm{O}$ co-solvents within several hours. However, upon sonication, the gel was formed within 4-5 minutes, and the critical gelling concentration also decreased considerably. The $\mathrm{L}^{-} \mathrm{Cl}^{-}$supramolecular gel exhibits helical fiber bundles or rope nanostructures. Careful analysis demonstrated that the helical fibers have $\mathrm{P}$ or $\mathrm{M}$ chirality randomly. Through a counter-anion exchange strategy, a chiral supra-amphiphile was prepared via triple hydrogen bonds between achiral $\mathrm{L}$ and a chiral menthylsulfate salt MS. Depending on the molecular chirality of sulfate, homochiral assembled nanotwists can be prepared, which was supported by SEM images and X-ray crystal structures. (+)-Menthylsulfate based supra-amphiphile L/(+)-MS self-assembled into uniform P-chirality nanotwists, while

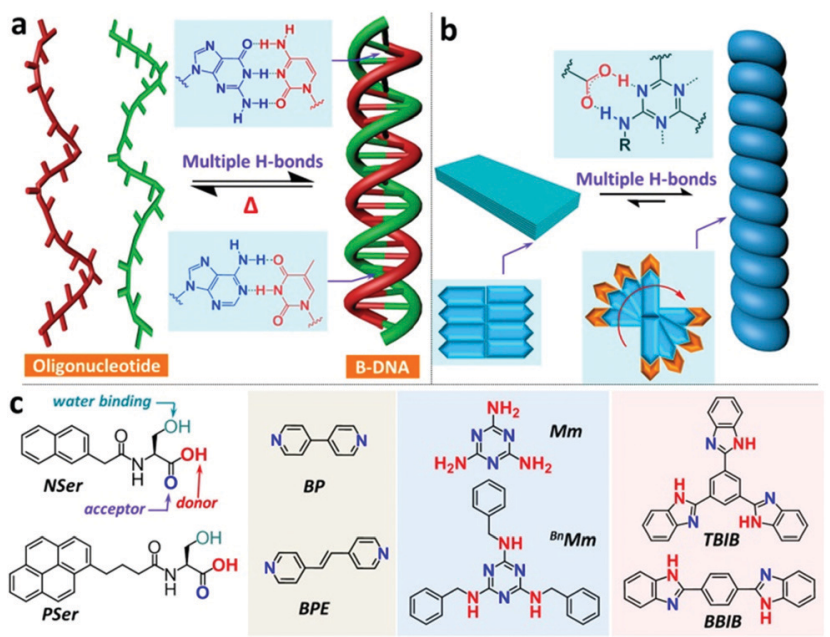

Fig. 8 Bioinspired formation of supra-amphiphiles via multiple hydrogen bonds. (a) Multiple hydrogen bonds in DNA. (b) Duplex hydrogen bonds among pyridine-based binders and carboxylic acids contribute to the formation of chiral nanostructures. (c) Chemical structures of aromatic amino acids and binders. (Reprinted with permission from ref. 97. Copyright (C) 2019 American Chemical Society.)

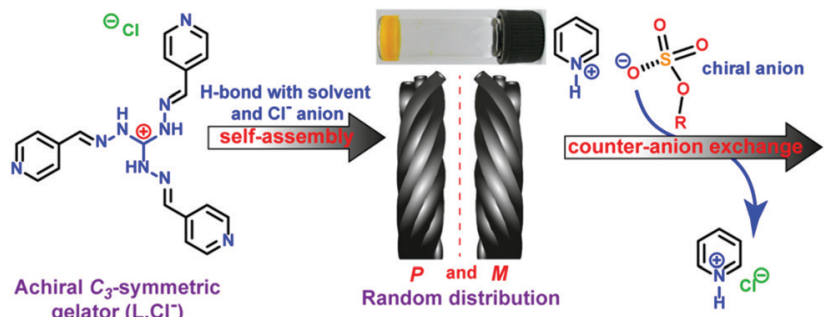
gelator (L.Cl`)

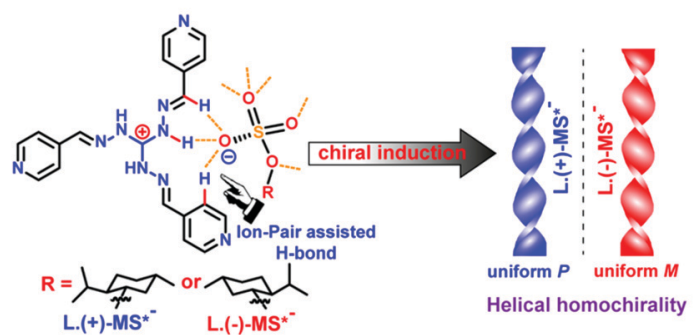

Fig. 9 Illustration of chemical structures and chirality control of selfassembled structures of supra-amphiphiles via the counter-anion exchange strategy. (Reprinted with permission from ref. 98. Copyright (C) 2016 American Chemical Society.)

(-)-menthylsulfate based supra-amphiphile L/(-)-MS gave uniform M-chirality nanotwists. This work showed that through the formation of chiral supra-amphiphiles via the counter-anion exchange method, the helical handedness of the assembly could be efficiently biased. ${ }^{98}$

\subsection{Self-assembly of other non-covalent interaction based chiral supra-amphiphiles}

Coordination bonds are also regarded as one of the most important non-covalent interactions. They have been widely used to synthesize size and shape controlled metallocycles; some of them are supra-amphiphiles. For example, Stang's group designed a chiral metallacycle type supra-amphiphile. ${ }^{99}$ As shown in Fig. 10, alanine-based dipyridyl ligand 3 and an organic Pt(II) complex acceptor 4 or $\mathbf{5}$ could form rhomboids $\mathbf{1}$ and hexagons 2, respectively. The metallocycles $\mathbf{1}$ and $\mathbf{2}$ are typical supra-amphiphiles according to their definition. These metallocycles can easily self-assemble into nanospheres at low concentration, while at high concentration, chiral metallogels are prepared driven by several non-covalent interactions, such as $\pi-\pi$ stacking, H-bonding and hydrophobic interactions. SEM images revealed that the metallogels are composed of chiral nanofibers and the chirality sense is determined by the molecular chirality of alanine. This work described a novel approach for developing chiral metallocycles, which might help to develop functional gel materials with controllable dimensionality and chirality.

Lee and his colleagues designed two bent aromatic amphiphiles, which contain a hydrophilic dendron at the apex and an $m$-pyridine moiety at the terminal. ${ }^{100}$ As shown in Fig. 11, compound 1 could form zigzag $2 \mathrm{D}$ flat sheets via $\pi-\pi$ stacking interactions. When coordinating with $\mathrm{Ag}(\mathrm{I})$ ions, compound 1 changed into dimeric macrocycles at a lower concentration, 


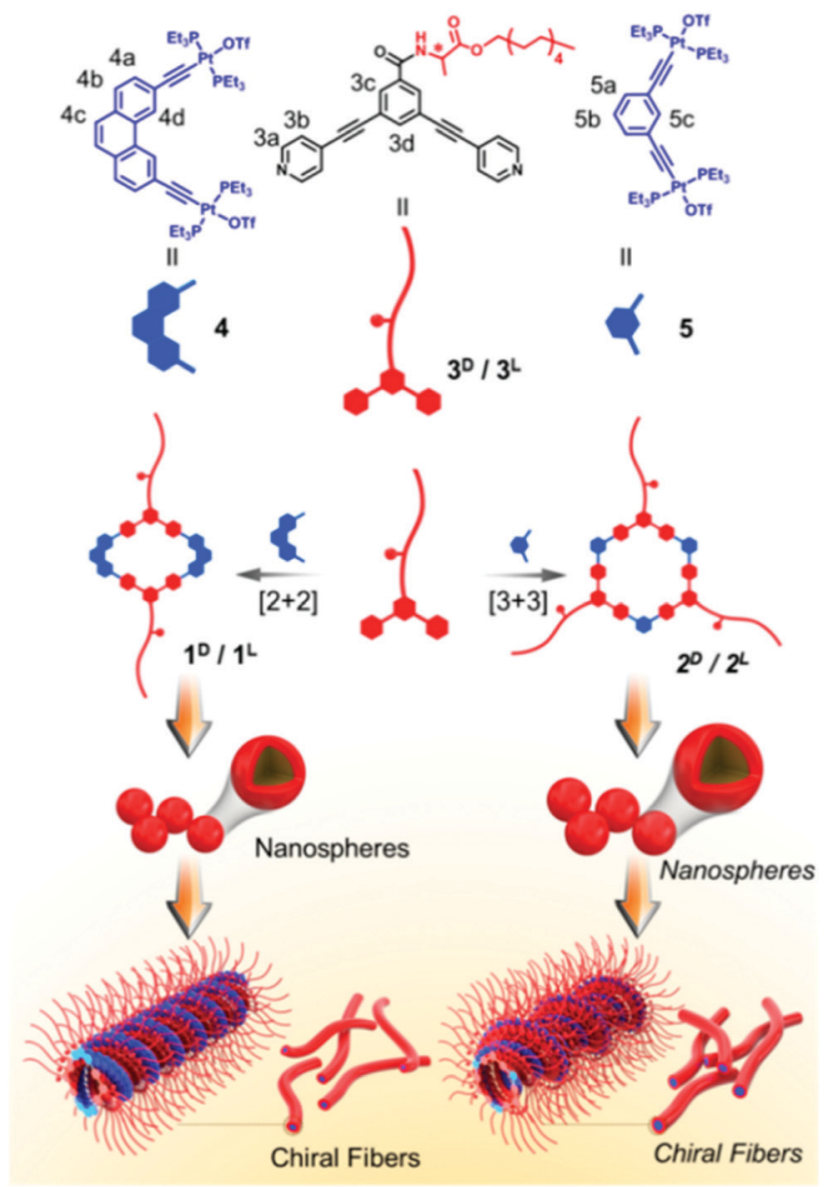

Fig. 10 Illustration of the chemical structures of building blocks and the formation and self-assembly of chiral metallocycles. (Reprinted with permission from ref. 99. Copyright (C) 2018 American Chemical Society.)

which is a typical chiral supra-amphiphile. Interestingly, at higher concentration, the dimeric macrocycles transformed into helical tubules with an internal cavity diameter of $2.5 \mathrm{~nm}$ and an external diameter of $7 \mathrm{~nm}$. CD spectra showed increased signals with increasing concentration, which indicated the helical stacking of dimeric macrocycles with a preferred handedness. These two states were reversible in response to concentration variation. Back-switching from helical tubules to flat sheets can be achieved by adding an excess amount of chloride salt, forming an $\mathrm{AgCl}$ precipitate. The results demonstrated that coordination could trigger drastic supramolecular switching between chiral helical tubules and achiral flat sheets.

Electrostatic interaction based chiral supra-amphiphiles have also been designed by several groups. For example, Oda and co-workers reported a series of cationic gemini achiral amphiphiles, which are based on ethelene-1,2-bis(dimethylalkylammonium) compounds. ${ }^{101}$ As shown in Fig. 12, these cationic achiral amphiphiles can form salts with several counterions of di-carboxylic acids, which are regarded as chiral supra-amphiphiles. When tartrate was used, twisted nanoribbons are obtained. Their width and pitch can be efficiently manipulated by the length of the hydrocarbon chain on the cationic part. Interesting, they found that when the counterion

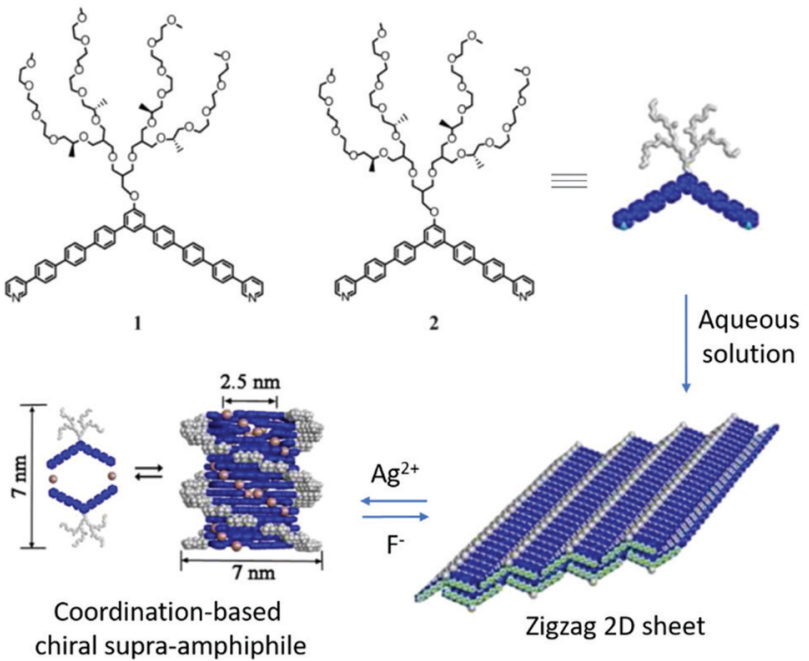

Fig. 11 Chemical structures of bent aromatic amphiphiles and illustration of the formation and self-assembly of coordination-triggered chiral supraamphiphiles. (Reprinted with permission from ref. 100. Copyright (C) 2013 American Chemical Society.)

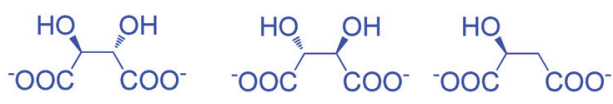

D-tartrate L-tartrate L-malate

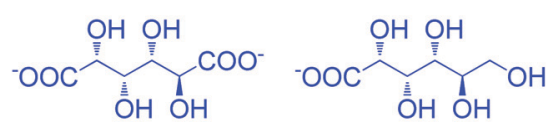

D-glucarate D-gluconate

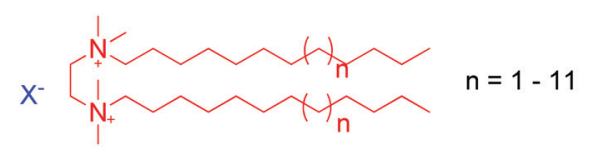

$\mathrm{X}^{-}=\mathrm{D}$-tartrate, L-tratrate, L-malate, D-glucarate, D-gluconate

Fig. 12 Illustration of electrostatic interaction based chiral supraamphiphiles from cationic achiral gemini surfactants and anion chiral carboxylic acid.

is malate, which only has one hydroxy group, the hydrogenbonding between the cation part and the anion part of the supra-amphiphile was weakened, leading to the formation of flat bilayers. When glucarate or gluconate are used as chiral counterions, although the hydrogen bonding remains, the binding affinity between the cation part and the anion part of the supra-amphiphile is weakened due to the larger distance between two carboxylic acids. They further build a model to explain the above experimental results. Their results contribute much to the understanding of the role of the chiral bilayer in the hierarchical formation of helical nanostructures, where the bilayer is constructed by the supra-amphiphile between achiral gemini and chiral counterions.

Chiral polyoxometalates (POMs) are difficult to synthesize for the following two possible reasons. Firstly, their topologic structure is very complex. Secondly, the dynamic exchange of 


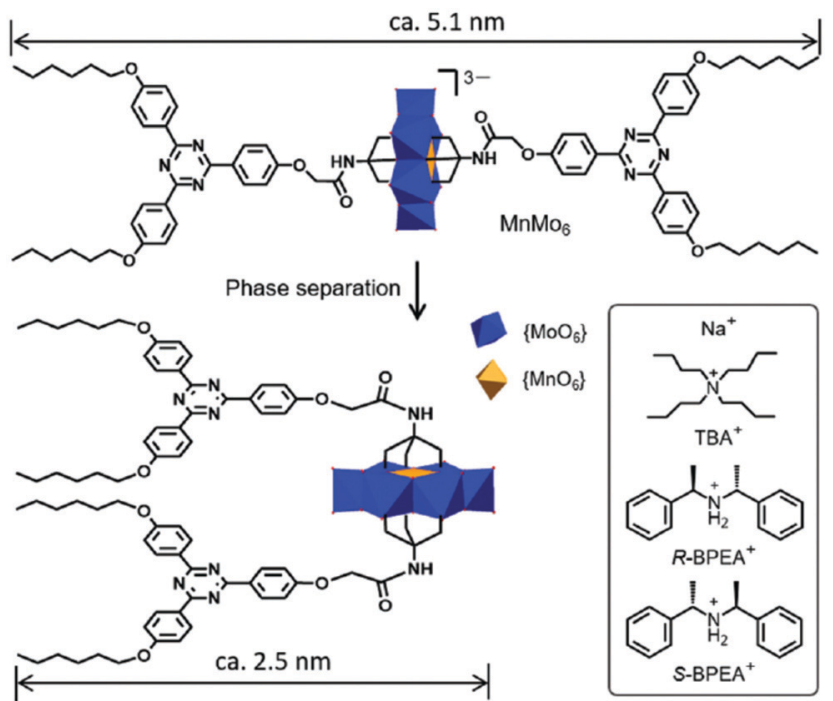

Fig. 13 Illustration and chemical structures of chiral POMs. (Reprinted with permission from ref. 102. Copyright (C) 2019 Royal Society of Chemistry.)

the oxygen molecules in the POMs with those in water leads to quick chirality disappearance. By designing an electrostatic interaction based chiral supra-amphiphile, Li and co-workers successfully reported a chiral POM and its chiral self-assembly in mixed solvents. ${ }^{102}$ As shown in Fig. 13, they prepared an Anderson-type POM, which contains a triazine derivative with two short alkoxy chains. By using two enantiomers of chiral bis(1-phenylethyl)ammonium, $R$-BPEA and $S$-BPEA, as the countercations, the morphologies of the POMs changed significantly. Through the observation of TEM images, they found that M- and P-type helical fibers existed as the self-assemblies of these two chiral POMs, which was further supported by the mirror-image CD signals. This work brings a new method for the design of chiral POMs by using the strategy of chiral supra-amphiphiles.

\subsection{Self-assembly of cyclodextrin based chiral supra-amphiphiles}

Host-guest interactions occupy a particular role in the development of supramolecular chemistry. Supra-amphiphiles based on such interactions have been well summarized in several reviews. For example, in 2015, Huang and co-workers systematically organised and analysed the formation, characterization, assembly and application of a series of supra-amphiphiles based on typical macrocyclic compounds including crown ethers, cyclodextrin, calixarene, cucurbituril and pillar $[n]$ arene. ${ }^{60}$ However, the chiral assembly of these supra-amphiphiles and their chirality-related functions are less discussed.

Cyclodextrin (CyD) is a well-known supramolecular host. $^{103-105}$ It consists of 6-8 D-glucose subunits joined by $\alpha-1,4$ glycosidic bonds, providing a chiral inner cavity, which can bind a chiral guest or an achiral guest to form chiral supraamphiphiles in water. In this subchapter, some recent examples of chiral self-assembly of cyclodextrin-based supra-amphiphiles will

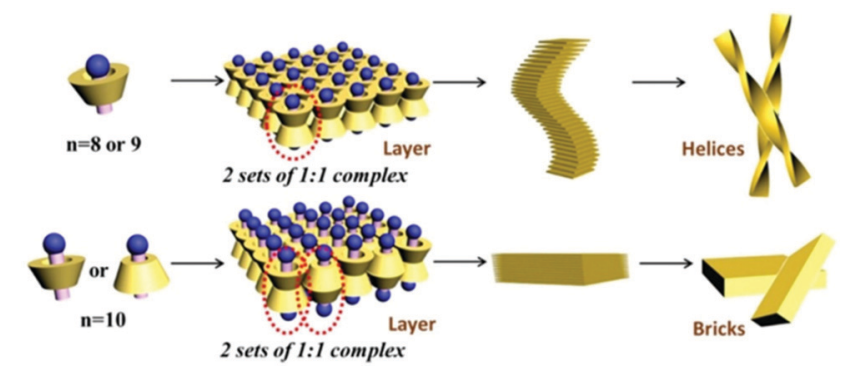

Fig. 14 Illustration of different complexation modes between long chain amines and $\beta$-CD. Upper: The amine head of $\mathrm{CH}_{3}\left(\mathrm{CH}_{2}\right)_{n-1} \mathrm{NH}_{2}(n=8,9)$ is toward the wider rim of $\beta$-cylcodextrin, which leads to the formation of helices. Bottom: The amine head of $\mathrm{CH}_{3}\left(\mathrm{CH}_{2}\right)_{n-1} \mathrm{NH}_{2}(n=10)$ is toward both the narrower and wider rims of $\beta$-cylcodextrin, which leads to the formation of planar bricks. (Reprinted with permission from ref. 106. Copyright (C) 2018 Wiley-VCH.)

be overviewed. We hope the analysis and summarization will contribute to further development of host-guest interaction based supra-amphiphiles in the chirality field (Fig. 14).

Huang, Yan ad co-workers reported a chiral supra-amphiphile based on $\beta$-cyclodextrin and a long alkyl amine. ${ }^{106}$ Under vortex mixing, achiral organic amines $\mathrm{CH}_{3}\left(\mathrm{CH}_{2}\right)_{n-1} \mathrm{NH}_{2}(n=6-10)$ were added into the saturated solution of $\beta$-cyclodextrin. For shorter chain amines of $\mathrm{CH}_{3}\left(\mathrm{CH}_{2}\right)_{n-1} \mathrm{NH}_{2}(n=6,7)$, NMR and FT-MS spectra indicated that a host-guest complex was not formed, which was also co-evidenced by no Tyndall effect. However, for long chain amines of $\mathrm{CH}_{3}\left(\mathrm{CH}_{2}\right)_{n-1} \mathrm{NH}_{2}(n=8,9,10)$, white precipitates were obtained, which were composed of mesoscopic right-handed helical ribbon structures $(n=8,9)$ and planar bulk bricks $(n=10)$. NMR titration experiments and FT-MS results supported a 1:1 host-guest ratio in all the systems. 2D-NMR experiments indicated that amines $\mathrm{CH}_{3}\left(\mathrm{CH}_{2}\right)_{n-1} \mathrm{NH}_{2}(n=8,9)$ were oriented threaded into the chiral cavity of $\beta$-CD.

They further measured the CD spectra and found different chiral signals depended on the length of the long chain amine. In the UV adsorption region of the head $\mathrm{NH}_{2}$ group, a positive CD signal was observed for $\mathrm{CH}_{3}\left(\mathrm{CH}_{2}\right)_{n-1} \mathrm{NH}_{2}(n=8)$, and a negative $\mathrm{CD}$ signal was observed for $\mathrm{CH}_{3}\left(\mathrm{CH}_{2}\right)_{n-1} \mathrm{NH}_{2}(n=9)$, while $\mathrm{CH}_{3}\left(\mathrm{CH}_{2}\right)_{n-1} \mathrm{NH}_{2}(n=10)$ was $\mathrm{CD}$ silent. According to Harata and Kodaka's rule, if a parallel electronic dipole is outside the cavity, it will give a negative induced chiral signal, otherwise it will be positive when inside the cavity. Combining these findings and the theory, they successfully controlled the induced chirality by changing the host-guest ratio. When increasing the mole ratio between $\beta$-CD and $\mathrm{CH}_{3}\left(\mathrm{CH}_{2}\right)_{n-1} \mathrm{NH}_{2}$ $(n=8)$ from $1: 1$ to $1: 4$, the CD signal was totally reversed from a positive signal to a negative one. Further adding more $\beta$-CD to the above system lead to the recovery of the CD signal to a positive one. Therefore, by simply tuning the dynamics of the host-guest interaction, the chirality-switching was well controlled.

Jiang and co-workers reported chiral supra-amphiphile SDS@2 $\beta$-CD based bio-mimetic lattice self-assembly. ${ }^{107}$ Despite big progress having been achieved in the preparation of crystalline structures from protein-mimetic molecules, some special 


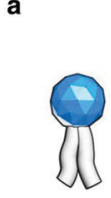

b
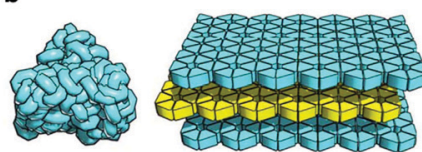

c

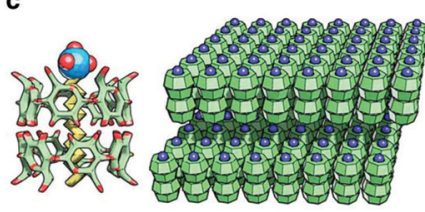

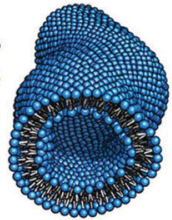
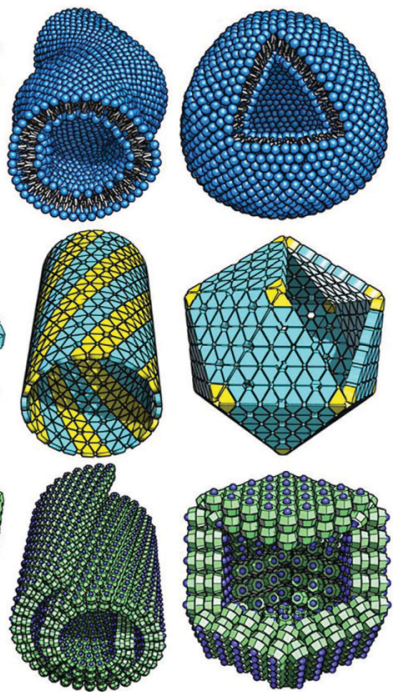

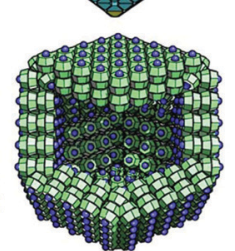

Fig. 15 Illustration of several self-assembly structures of (a) lipid molecules, (b) proteins and (c) SDS $a 2 \beta-C D$ supra-amphiphiles. (Reprinted with permission from ref. 107. Copyright (C) 2017 Springer Nature.)

capsid-like structures such as hollow rhombic dodecahedra and helical tubes are still a fundamental challenge. In their report, as shown in Fig. 15, the SDS@2 $\beta$-CD complex can form polyhedral (4-6 wt\%), tubular (6-25 wt\%) and lamellar (25-50 wt\%) phases depending on its concentration. Dodecahedral structures were observed for the first time in a selfassembly system, whose size is comparable to and even larger than the largest known virus in nature. These self-assemblies were similar to those from lipid molecule and protein assembly, thus providing a simple design rule for the design of new proteinmimetic materials.

Liu and colleagues designed an azobenzene-containing supra-dendron, which is a host-guest interaction based supra-amphiphile. ${ }^{108}$ As shown in Fig. 16, the azobenzene moiety is covalently linked with a glutamic acid dendron with a short alkyl chain. The dendron amphiphile was abbreviated as $\mathbf{A Z O C}_{\mathbf{8}} \mathbf{G A c}$, which self-assembled into a hydrogel via a heating-cooling procedure. A reversible macroscopic volume phase transition of the gel was observed in response to UV irradiation and $\mathrm{pH}$ variations. Although the UV-vis spectrum showed complete reversibility during this process, the chiroptical property, which is characterized by circular dichroism, showed incomplete reversibility. The authors prepared the inclusion complex $\mathrm{CD} / \mathbf{A Z O C}_{8} \mathbf{G A c}$ with $\alpha, \beta, \gamma$-cyclodextrins. Isothermal titration calorimetry (ITC) experiments indicated that the $\alpha-\mathrm{CD} / \mathbf{A Z O C}_{\mathbf{8}} \mathbf{G A c}, \beta-\mathrm{CD} / \mathbf{A Z O C}_{8} \mathbf{G A c}$, and $\gamma-\mathrm{CD} / \mathbf{A Z O C} \mathbf{C}_{8} \mathbf{G A c}$ gave a $1: 1,5: 4$ and $2: 1$ host/guest ratio, respectively. However, $\alpha-\mathbf{C D} / \mathbf{A Z O C}_{8} \mathbf{G A c}$ had a bigger binding constant compared with its analogues $\beta$-CD and $\gamma$-CD, indicating the formation of a more stable inclusion complex. The supra-dendron $\alpha-\mathrm{CD} /$ $\mathbf{A Z O C}_{8} \mathbf{G A c}$ can also form a hydrogel, which was composed of nanofibers as demonstrated by AFM images. It was found that enhanced chiroptical switching capacity was successfully realized.

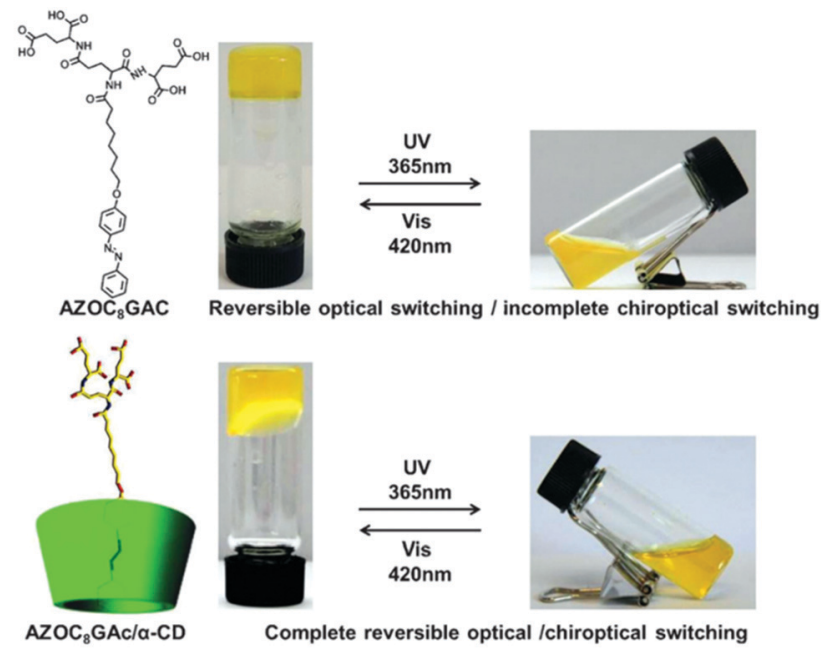

Fig. 16 Molecular structures of the dendron gelator and cyclodextrin based supra-dendron. Photographs of the gels showed a reversible gel-sol transition. (Reprinted with permission from ref. 108. Copyright (C) 2016 Wiley-VCH.)

Liu and Duan recently designed a pyrene-dendron based supra-amphiphile and investigated its self-assembly as well as its circularly polarized luminescence (CPL) properties. ${ }^{109}$ As shown in Fig. 17, the individual pyrene-dendron, which is named PGAc, can self-assemble into a hydrogel at varied $\mathrm{pH}$ values from 3-12. AFM images revealed that the hydrogels were composed of chiral nanohelices. Interestingly, the pitches of the helices were related to the $\mathrm{pH}$ values. When increasing and lowering the $\mathrm{pH}$, the length of the pitch gradually increased and decreased, respectively. This system might be useful for
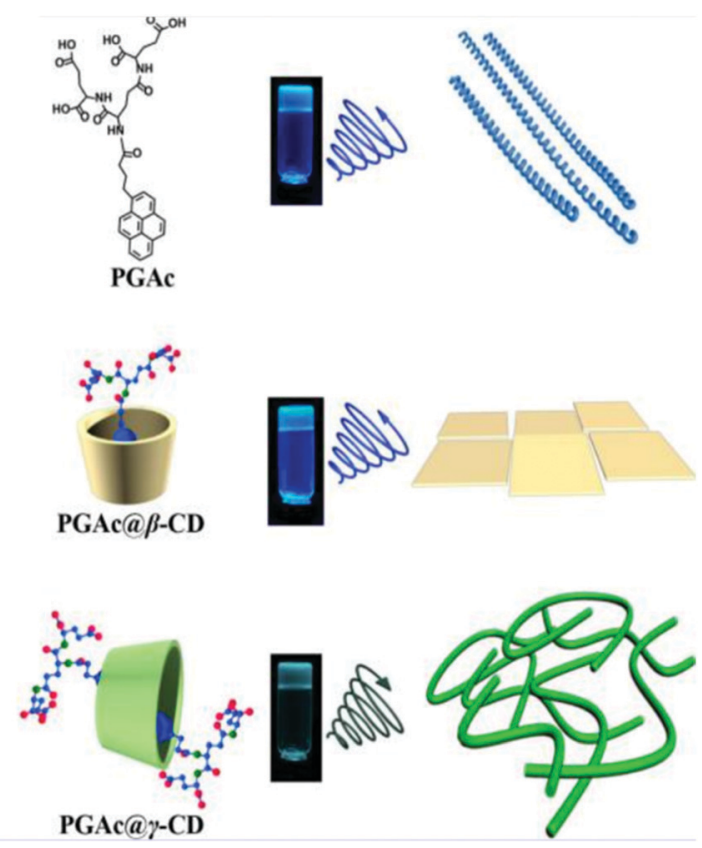

Fig. 17 Molecular structures and illustration of supra-amphiphiles. The right column shows the nanostructures of supra-gels. (Reprinted with permission from ref. 109. Copyright (C) 2018 American Chemical Society.) 
the preparation of $\mathrm{pH}$-regulated smart springs. They further prepared a cyclodextrin based host-guest complex PGAc/CyD. It was found that the CPL spectrum of PGAc/ $\beta$-CyD ascribed to monomer emission showed a blue shift from $475 \mathrm{~nm}$ to $440 \mathrm{~nm}$ compared with the PGAc hydrogel. However, the PGAc $/ \gamma-\mathrm{CyD}$ inclusion complex showed excimer emission at $480 \mathrm{~nm}$, which indicated strong $\pi-\pi$ stacking of two pyrenes. CPL spectra showed positive and negative signals corresponding to monomer and excimer emission of pyrenes, respectively. Moreover, $\alpha$-CyD showed no host-guest interaction with PGAc, which is confirmed by CPL and fluorescence spectra. This strategy can be used to modify the self-assembly and chiroptical properties of supramolecular hydrogelators.

Stimuli-responsive circularly polarized luminescence materials have attracted a lot of attention recently. By incorporating a cyanostilbene conjugated hydrogelator into the inner cavity of cyclodextrin to form a supra-gelator, Liu and co-workers successfully constructed a photo-regulated reversible chiroptical gel from a $\gamma$-CyD supra-gelator, which showed enhanced circularly polarized luminescence. The hydrogelator, abbreviated as CG, could form helical nanotubes via the gelation process. ${ }^{110}$ However, the CG hydrogel showed no responsiveness under photo irradiation, which is probably due to the compact stacking of the cyano-stilbene moiety. To overcome this problem, they used cyclodextrins as hosts to encapsulate the CG via the inner cavity of CyD, which can provide enough space for the photo reaction of cyano-stilbene. By mixing CyD and CG, a 1:1 host-guest complex was obtained for all the three cyclodextrins, $\alpha-\mathrm{CyD}, \beta-\mathrm{CyD}$ and $\gamma$-CyD, which were named supra-gelators and belonged to supraamphiphiles. These supra-gelators could also form hydrogels, which were composed of helical nanotubes as demonstrated by SEM and TEM images, similar to those self-assembled from the individual CG. Although the $\alpha$-CyD and $\beta$-CyD host-guest interaction supra-gelator showed no response to photoirradiation, the $\gamma$-CyD supra-hydrogel showed excellent photo-responsiveness, giving reversible CD, CPL spectra and morphological changes of the assemblies. Its CD and CPL signals gradually disappeared under photo-irradiation. After a heating-cooling procedure, these chiral signals could be successfully recovered. Besides, the morphology showed complete reversibility between helical nanotubes and nanospheres. By designing a series of control experiments, such as rheology measurements, binding constant measurements and FT-IR experiments, they explained that the larger inner cavity, low mechanical strength and small binding constant might lead to excellent photo-reversibility for the $\gamma$-CyD/CG supra-gelator.

CyD is a chiral host from D-sugar, while CG is derived from glutamic acid and thus $\mathrm{L}$ and D-enantiomers were obtained. Both CG enantiomers could form host-guest interaction supragelators with CyD. Interestingly, the helicity of the selfassembled nanotubes was the same as that of $\mathrm{L}^{-}$or D-CG, which indicated that the chirality of cyclodextrin has no effect on the whole system. However, they found that the supramolecular chirality and circularly polarized luminescence were obviously enhanced compared with that of individual CG. These results demonstrated that CyD cannot control the chirality sign but enhance the chirality intensity of the supra-hydrogels, which

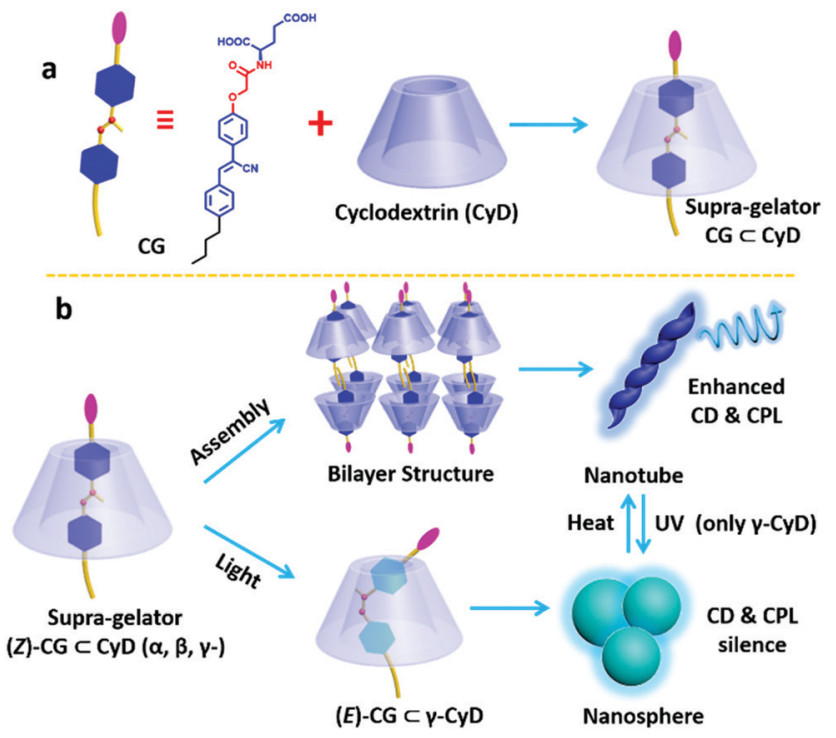

Fig. 18 Schematic representation of the formation of supra-amphiphiles and the reversible transformation of the self-assembly route as well as the nanostructures and the molecular structure of guest molecule CG and the dendron gelator and supra-dendron. Photographs of the gels showed a reversible gel-sol transition. (Reprinted with permission from ref. 111. Copyright (C) 2019 Royal Society of Chemistry.)

was an interesting phenomenon that has not been reported before (Fig. 18). ${ }^{111}$

By forming a three-component supra-amphiphile using cyclodextrin, an azobenzene-modified Anderson-type polyoxometalate (POM), and positively-charged methylene blue (MB) dye, $\mathrm{Wu}$ and co-workers realized chiral transfer and amplification to achiral dyes from cyclodextrin under the cooperation of host-guest interactions and electrostatic interactions. ${ }^{112}$ As illustrated in Fig. 19, the single-side-modified POM is synthesized in tetrabutyl ammonium salt, which successfully gave single crystals in water/ethanol co-solvents at $\mathrm{pH}$ 5. After forming the host-guest complex, the Azo-POM/CyD system was examined using circular dichroism spectra. Both $\alpha$-CyD and $\beta$-CyD including POMs showed obviously negative and positive Cotton signals with a crossover at about $380 \mathrm{~nm}$, ascribed to the azobenzene group on the POMs. However, the $\gamma$-CyD/POM hybrid system gave no CD signal. These results indicated that the molecular chirality of $\alpha$ - and $\beta$-CyDs could be successfully transferred to Azo-POM. When further adding positively charged dye MB, new positive CD signals at $558 \mathrm{~nm}$ and $720 \mathrm{~nm}$

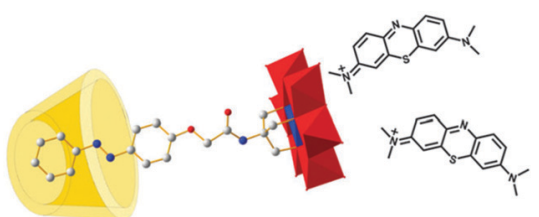

Fig. 19 Schematic drawing of the Azo-POM bridged three component supra-amphiphile, the yellow barrel-shaped part represents cyclodextrin, the ball-and-stick part represents Azo-POM. (Reprinted with permission from ref. 112. Copyright (C) 2014 Royal Society of Chemistry.) 
and a strong negative Cotton signal at $629 \mathrm{~nm}$ appeared, which was due to the induced chirality of the MB dye. For better understanding of chirality transfer, control experiments were also designed. No CD signals were found when Azo-POM was mixed with MB. When $\alpha$-CD was mixed with MB, chiral signals belonging to $\mathrm{MB}$ could still not be observed. These results demonstrated that the chirality of cyclodextrin can only be transferred to MB by bridged Azo-POM through the formation of the three-component supra-amphiphile, which provides useful insight in developing POM bridged chiral supramolecular systems.

$\mathrm{Li}$, Tian and co-workers designed an axially chiral 1,1'-binaphthyl compound containing a photo-responsive azobenzene and a hydrophilic 4,4'-bipyridinium moiety. This compound can form a typical two chain supra-amphiphile with two equivalents of $\alpha$-cyclodextrin. ${ }^{113}$ As shown in Fig. 20, the synthesized supra-amphiphile can be further used as a monomer to form a supramolecular polymer with a bola-type host bis( $p$-sulfonatocalix[4]-arene). The formation of the supra-amphiphile was well confirmed by ${ }^{1} \mathrm{H}$ NMR spectroscopy, while the formation of the supramolecular polymer was demonstrated by UV-vis adsorption and circular dichroism spectra. UV-vis spectra indicated that the absorption band of the supra-amphiphile was similar to that of monomer $\mathrm{M}$, which meant that the chiral cyclodextrin did not influence the absorption of the chromophores. For the supramolecular polymer, its absorption curve is almost the overlap of the supra-amphiphile and host bis( $p$-sulfonatocalix[4]-arene), which indirectly supports its formation. They also measured DLS, SEM, AFM and TEM data to support the formation of the supramolecular polymer. More interestingly, the self-assembly pathway can be photo-regulated based on the trans-cis transformation of the azobenzene moiety. This work presents a perfect example of the cooperation of the concepts of supra-amphiphiles and supramolecular polymerization.

\subsection{Self-assembly of in situ formed cyclodextrin based chiral supra-amphiphiles at the air/water interface}

By utilizing Langmuir-Blodgett technology, Liu and co-workers reported an in situ formed chiral supra-amphiphile based on host-guest interactions at the air/water interface. ${ }^{114}$ They designed and synthesized a glutamic acid dendron amphiphile containing an azobenzene moiety at the amine head, which was abbreviated as AzoGE. As shown in Fig. 21, this compound could self-assemble into a stable monolayer at the air/water interface due to the good equilibrium of hydrophilic and hydrophobic abilities, which could be easily transferred onto a solid substrate for further AFM observation and other measurements. AFM images indicated that uniform nanofibers were formed and an exciton couplet was found in the CD spectrum at the azobenzene absorption region, which proved successful chirality transfer from glutamic acid to the achiral azobenzene moiety. However, the film made from the air/ aqueous CyD system showed circular domains, which exhibited a negative Cotton effect. These results indicated that the in situ formation of supra-amphiphiles via host-guest interactions at air/water interface could significantly manipulate the self-assembled

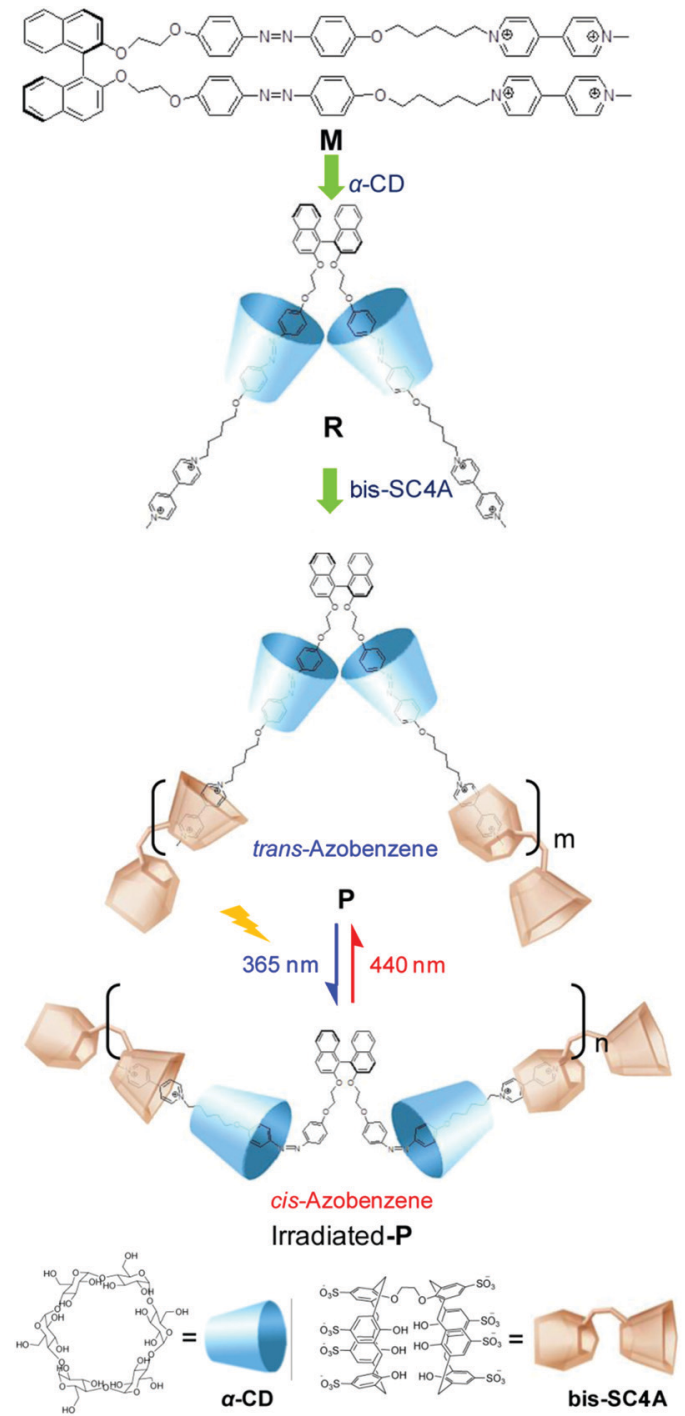

Fig. 20 Molecular structures of the dendron gelator and supra-dendron. Photographs of the gels showed a reversible gel-sol transition. (Reprinted with permission from ref. 113. Copyright (C) 2013 American Chemical Society.)

nanostructures and supramolecular chirality. Most importantly, the nanofiber film from the water subphase showed no obvious response to UV irradiation, although the azobenzene moiety has a well-known cis-trans configuration transformation. However, the film from the $\alpha$-CyD subphase showed impressive reversible optical and chiroptical properties.

Liu and co-workers also designed an anthracene functionalized glutamic acid dendron, which was named AGE. AGE could form a stable monolayer using Langmuir-Blodgett technology at the air/water interface. As shown in Fig. 22, when adding cyclodextrin to the aqueous phase, AGE could be incorporated into the inner cavity of $\gamma$-CyD to form a chiral supra-amphiphile in situ at the interface. ${ }^{115}$ However, this supra-amphiphile was unstable and increasing the surface pressure led to its disassembly. Due to the photo-dimerization of anthracene, the bola-type supra-amphiphile could be fixed to form a stable 


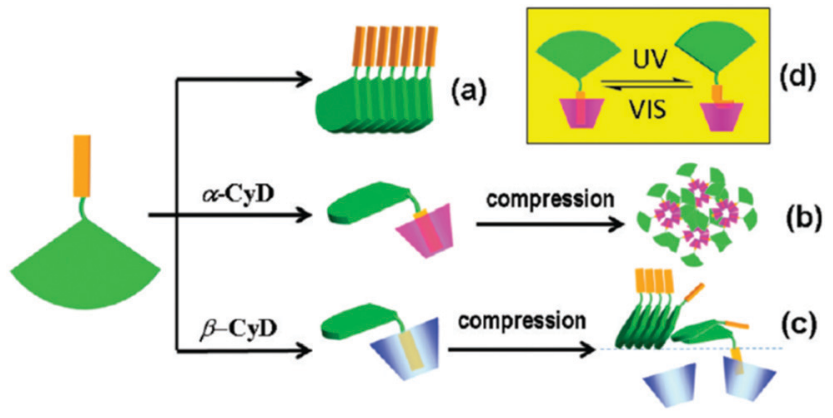

Fig. 21 Illustration of the formation of cyclodextrin based supraamphiphiles at the air/water interface using Langmuir-Blodgett technology. (a) Stacking mode of AzoGE. (b) Formation of the supra-amphiphile based on $\alpha-C y D$, further compression promotes aggregation. (c) Formation of the supra-amphiphile based on $\beta$-CyD, further compression destroyed the supra-amphiphile. (d) cis-trans transformation of azobenzene. (Reprinted with permission from ref. 114. Copyright (C) 2011 American Chemical Society.)

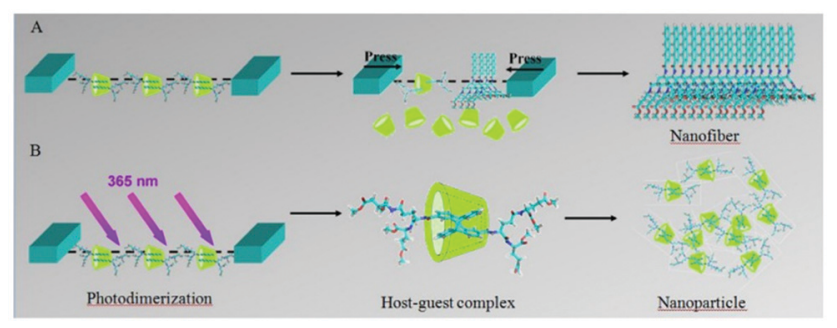

Fig. 22 Illustration of self-assembly of in situ supra-amphiphiles at the air/ water interface. (A) The AGE/ $\gamma$-CyD supra-amphiphile decomposed under larger surface pressure, AGE individually self-assembled into nanofibers. (B) UV $365 \mathrm{~nm}$ irradiation led to the dimerization of anthracene, the supraamphiphile further formed a very stable rotaxane supra-amphiphile, which self-assembled into nanoparticles under larger surface pressure. (Reprinted with permission from ref. 115. Copyright (C) 2014 Elsevier.)

rotaxane-like complex, which could be regarded as a new supraamphiphile. The rotaxane supra-amphiphile was further pushed together to form nanoparticles under compression at the air/water interface. This work provided a useful method for in situ construction of stable supra-amphiphiles based on cyclodextrins.

\section{Conclusions and perspectives}

Here, we summarized the recent progress of the design and self-assembly of chiral supra-amphiphiles, which are formed mainly via hydrogen bonds and host-guest interactions. In general, two main strategies can be adopted to design chiral supra-amphiphiles, one is that both the hydrophobic moiety and hydrophilic moiety are chiral, and the other is that only one of them is chiral. Through self-assembly, highly ordered chiral nano- or micro-structures can be prepared from these supra-amphiphiles, which showed appealing applications in many fields, such as chiroptical switches, chiral recognition, chiral catalysis and chiral luminescent materials. Although some achievements have been made with these chiral supra-amphiphiles, it is clear that chiral self-assembly could further be expanded to more complex systems with the concept of supra-amphiphiles. There is still plenty of room to establish exquisite chiral supraamphiphiles based on varied non-covalent interactions and a variety of combinations of molecules. Here, we only limited host-guest interactions to the cyclodextrins. Many more hosts can be used to construct chiral supra-amphiphiles and thus their nanoassemblies. In addition, many biological molecules could be used as one chiral component of supra-amphiphiles and their self-assembly as well as new functions are expected. From the view of functions, asymmetric catalysis and enzyme mimics could be challenging and interesting.

\section{Conflicts of interest}

There are no conflicts to declare for the authors.

\section{Acknowledgements}

The authors are grateful for the financial support from the Chinese Academy of Sciences (QYZDJSSW-SLH044), National Natural Science Foundation of China (21890734, 21861132002, 21602223) and Youth Innovation Promotion Association of CAS (2019036).

\section{References}

1 S. Saha, B. Holzapfel, Y.-T. Chen, K. Terlinden, P. Lill, C. Gatsogiannis, H. Rehage and G. H. Clever, J. Am. Chem. Soc., 2018, 140, 17384-17388.

2 A. Wang, L. Cui, S. Debnath, Q. Dong, X. Yan, X. Zhang, R. V. Ulijn and S. Bai, ACS Appl. Mater. Interfaces, 2017, 9, 21390-21396.

3 L. K. Solomon, J. B. Kronenberg and H. C. Fry, J. Am. Chem. Soc., 2017, 139, 8497-8507.

4 C. Diaferia, F. A. Mercurio, C. Giannini, T. Sibillano, G. Morelli, M. Leone and A. Accardo, Sci. Rep., 2016, 6, 26638.

5 K. Jie, Y. Zhou, Y. Yao and F. Huang, Chem. Soc. Rev., 2015, 44, 3568-3587.

6 S. Fleming and R. V. Ulijn, Chem. Soc. Rev., 2014, 43, 8150-8177.

7 X. Mulet, C. E. Conn, C. Fong, D. F. Kennedy, M. J. Moghaddam and C. J. Drummond, Acc. Chem. Res., 2013, 46, 1497-1505.

8 M. Kwak and A. Herrmann, Chem. Soc. Rev., 2011, 40, 5745-5755.

9 X. Zhao, F. Pan, H. Xu, M. Yaseen, H. Shan, C. A. E. Hauser, S. Zhang and J. R. Lu, Chem. Soc. Rev., 2010, 39, 3480-3498.

10 R. Srinivas, S. Samanta and A. Chaudhuri, Chem. Soc. Rev., 2009, 38, 3326-3338.

11 T. L. Greaves and C. J. Drummond, Chem. Soc. Rev., 2008, 37, 1709-1726.

12 L. van t'Hag, S. L. Gras, C. E. Conn and C. J. Drummond, Chem. Soc. Rev., 2017, 46, 2705-2731. 
13 D. Lombardo, M. A. Kiselev, S. Magazu and P. Calandra, Adv. Condens. Matter Phys., 2015, 151683.

14 E. De Santis and M. G. Ryadnov, Chem. Soc. Rev., 2015, 44, 8288-8300.

15 J. Zhang, L. Peng and B. Han, Soft Matter, 2014, 10, 5861-5868.

16 A. Sorrenti, O. Illa and R. M. Ortuno, Chem. Soc. Rev., 2013, 42, 8200-8219.

17 T. L. Greaves and C. J. Drummond, Chem. Soc. Rev., 2013, 42, 1096-1120.

18 C. Fong, T. Le and C. J. Drummond, Chem. Soc. Rev., 2012, 41, 1297-1322.

19 S. Maurer, Life, 2017, 7, 44.

20 M. T. Jeena, L. Palanikumar, E. M. Go, I. Kim, M. G. Kang, S. Lee, S. Park, H. Choi, C. Kim, S.-M. Jin, S. C. Bae, H. W. Rhee, E. Lee, S. K. Kwak and J.-H. Ryu, Nat. Commun., 2017, 8, 26.

21 A. Sadaf, K. H. Cho, B. Byrne and P. S. Chae, in Membrane Proteins - Engineering, Purification and Crystallization, ed. A. K. Shukla, 2015, vol. 557, pp. 57-94.

22 Z. Wang, X. Hou, J. Xu, L. Shi and H. Liu, Pet. Sci. Technol., 2017, 35, 1130-1134.

23 M. Das, Y. Du, J. S. Mortensen, H. E. Bae, B. Byrne, C. J. Loland, B. K. Kobilka and P. S. Chae, Chem. - Eur. J., 2018, 24, 9860-9868.

24 K. H. Cho, P. Hariharan, J. S. Mortensen, Y. Du, A. K. Nielsen, B. Byrne, B. K. Kobilka, C. J. Loland, L. Guan and P. S. Chae, ChemBioChem, 2016, 17, 2334-2339.

25 S. Polarz, J. A. Odendal, S. Hermann and A. Klaiber, Curr. Opin. Colloid Interface Sci., 2015, 20, 151-160.

26 P. S. Chae, H. E. Bae, M. Ehsan, H. Hussain and J. W. Kim, Org. Biomol. Chem., 2014, 12, 8480-8487.

27 T. Xiao, W. Zhong, L. Xu, X.-Q. Sun, X.-Y. Hu and L. Wang, Org. Biomol. Chem., 2019, 17, 1336-1350.

28 P. Li, Y. Chen and Y. Liu, Chin. Chem. Lett., 2019, 30, 1190-1197.

29 A. Jana, S. Bahring, M. Ishida, S. Goeb, D. Canevet, M. Salle, J. O. Jeppesen and J. L. Sessler, Chem. Soc. Rev., 2018, 47, 5614-5645.

30 S. Debnath, S. Roy and R. V. Ulijn, J. Am. Chem. Soc., 2013, 135, 16789-16792.

31 J. D. Badjic, A. Nelson, S. J. Cantrill, W. B. Turnbull and J. F. Stoddart, Acc. Chem. Res., 2005, 38, 723-732.

32 H. Su, W. Zhang, H. Wang, F. Wang and H. Cui, J. Am. Chem. Soc., 2019, 141, 11997-12004.

33 K. Sato, M. P. Hendricks, L. C. Palmer and S. I. Stupp, Chem. Soc. Rev., 2018, 47, 7539-7551.

34 A. J. Savyasachi, O. Kotova, S. Shanmugaraju, S. J. Bradberry, G. M. O'Maille and T. Gunnlaugsson, Chem, 2017, 3, 764-811.

35 A. Winter and U. S. Schubert, Chem. Soc. Rev., 2016, 45, 5311-5357.

36 T. Aida, E. W. Meijer and S. I. Stupp, Science, 2012, 335, 813-817.

37 W. Tao, Y. Liu, B. Jiang, S. Yu, W. Huang, Y. Zhou and D. Yan, J. Am. Chem. Soc., 2012, 134, 762-764.
38 D. Jiao, J. Geng, X. J. Loh, D. Das, T.-C. Lee and O. A. Scherman, Angew. Chem., Int. Ed., 2012, 51, 9633-9637.

39 X. Zhang and C. Wang, Chem. Soc. Rev., 2011, 40, 94-101.

40 W. An, H. Zhang, L. Sun, A. Hao, J. Hao and F. Xin, Carbohydr. Res., 2010, 345, 914-921.

41 X. Yu, C. Tu, L. He, R. Wang, G. Sun, D. Yan and X. Zhu, J. Macromol. Sci., Part A: Pure Appl. Chem., 2009, 46, 360-367.

42 S.-P. Wang, W. Lin, X. Wang, T.-Y. Cen, H. Xie, J. Huang, B.-Y. Zhu, Z. Zhang, A. Song, J. Hao, J. Wu and S. Li, Nat. Commun., 2019, 10, 1399.

43 J. Li, H. Li, J. Wang, H. Jiang, C. Yao, G. Wang, H. Ma and L. Shi, J. Surfactants Deterg., 2019, 22, 209-216.

44 G. Wang, J. Sun, L. An and S. Liu, Biomacromolecules, 2018, 19, 2542-2548.

45 C. Wang, Z. Wang and X. Zhang, Acc. Chem. Res., 2012, 45, 608-618.

46 J. Wang, X. Ding and X. Guo, Adv. Colloid Interface Sci., 2019, 269, 187-202.

47 X. Lv, D. Xia, Y. Zuo, X. Wu, X. Wei and P. Wang, Langmuir, 2019, 35, 8383-8388.

48 Y. Zhou, E. Li, R. Zhao and K. Jie, Org. Lett., 2018, 20, 4888-4892.

49 Y. Zhou, K. Jie and F. Huang, Chem. Commun., 2018, 54, 12856-12859.

50 D. Wu, Y. Li, J. Shen, Z. Tong, Q. Hu, L. Li and G. Yu, Chem. Commun., 2018, 54, 8198-8201.

51 Y. Zhou, K. Jie and F. Huang, Org. Chem. Front., 2017, 4, 2387-2391.

52 G. Yu, W. Yu, L. Shao, Z. Zhang, X. Chi, Z. Mao, C. Gao and F. Huang, Adv. Funct. Mater., 2016, 26, 8999-9008.

53 X. Chi, H. Zhang, G. I. Vargas-Zuniga, G. M. Peters and J. L. Sessler, J. Am. Chem. Soc., 2016, 138, 5829-5832.

54 Y. Yao, X. Chi, Y. Zhou and F. Huang, Chem. Sci., 2014, 5, 2778-2782.

55 Y. Kang, K. Liu and X. Zhang, Langmuir, 2014, 30, 5989-6001.

56 G. Yu, J. Li, W. Yu, C. Han, Z. Mao, C. Gao and F. Huang, Adv. Mater., 2013, 25, 6373-6379.

57 B. Jiang, W. Tao, X. Lu, Y. Liu, H. Jin, Y. Pang, X. Sun, D. Yan and Y. Zhou, Macromol. Rapid Commun., 2012, 33, 767-772.

58 H. Zhang, Z. Liu and Y. Zhao, Chem. Soc. Rev., 2018, 47, 5491-5528.

59 W. Shao, X. Liu, T. Wang and X.-Y. Hu, Chin. J. Org. Chem., 2018, 38, 1107-1118.

60 G. Yu, K. Jie and F. Huang, Chem. Rev., 2015, 115, 7240-7303.

61 H. Zhang, F. Xin, Y. Li, A. Hao, W. An and T. Sun, Prog. Chem., 2010, 22, 2276-2281.

62 Y. Chang, Y. Jiao, H. E. Symons, J.-F. Xu, C. F. J. Faul and X. Zhang, Chem. Soc. Rev., 2019, 48, 989-1003.

63 C. Wang, Z. Wang and X. Zhang, Small, 2011, 7, 1379-1383. 64 P. Xing, T. Sun and A. Hao, RSC Adv., 2013, 3, 24776-24793. 65 W. Ma, L. Xu, A. F. de Moura, X. Wu, H. Kuang, C. Xu and N. A. Kotov, Chem. Rev., 2017, 117, 8041-8093. 
66 L.-J. Chen, H.-B. Yang and M. Shionoya, Chem. Soc. Rev., 2017, 46, 2555-2576.

67 X. Zhang, J. Yin and J. Yoon, Chem. Rev., 2014, 114, 4918-4959.

68 Y. Yang, Y. Zhang and Z. Wei, Adv. Mater., 2013, 25, 6039-6049.

69 C. Wolf and K. W. Bentley, Chem. Soc. Rev., 2013, 42, 5408-5424.

70 J.-H. Xie, S.-F. Zhu and Q.-L. Zhou, Chem. Rev., 2011, 111, 1713-1760.

71 H. Qiu and S. Che, Chem. Soc. Rev., 2011, 40, 1259-1268.

72 X. Liu, L. Lin and X. Feng, Acc. Chem. Res., 2011, 44, 574-587.

73 L. A. P. Kane-Maguire and G. G. Wallace, Chem. Soc. Rev., 2010, 39, 2545-2576.

74 F. Ishiwari, Y. Shoji and T. Fukushima, Chem. Sci., 2018, 9, 2028-2041.

75 S. Basak, I. Singh, A. Ferranco, J. Syed and H.-B. Kraatz, Angew. Chem., Int. Ed., 2017, 56, 13288-13292.

76 C. Liu, D. Yang, Q. Jin, L. Zhang and M. Liu, Adv. Mater., 2016, 28, 1644-1649.

77 O. A. Bell, G. Wu, J. S. Haataja, F. Broemmel, N. Fey, A. M. Seddon, R. L. Harniman, R. M. Richardson, O. Ikkala, X. Zhang and C. F. J. Faul, J. Am. Chem. Soc., 2015, 137, 14288-14294.

78 T. G. Barclay, K. Constantopoulos, W. Zhang, M. Fujiki, N. Petrovsky and J. G. Matisons, Langmuir, 2013, 29, 10001-10010.

79 Y. Huang and Z. Wei, Chin. Sci. Bull., 2012, 57, 4246-4256.

80 L. Hsu, G. L. Cvetanovich and S. I. Stupp, J. Am. Chem. Soc., 2008, 130, 3892-3899.

81 E. A. Dennis, J. Biol. Chem., 2016, 291, 24431-24448.

82 Z. C. Tu and U. Seifert, Phys. Rev. E: Stat., Nonlinear, Soft Matter Phys., 2007, 76, 031603.

83 M. Liu, L. Zhang and T. Wang, Chem. Rev., 2015, 115, 7304-7397.

84 L. C. Palmer and S. I. Stupp, Acc. Chem. Res., 2008, 41, 1674-1684.

85 A. Sikder, D. Ray, V. K. Aswal and S. Ghosh, Angew. Chem., Int. Ed., 2019, 58, 1606-1611.

86 S. Scheiner, Struct. Chem., 2019, 30, 1119-1128.

87 J. Yao, C. Yu, Z. Liu, H. Luo, Y. Yang, G. Zhang and D. Zhang, J. Am. Chem. Soc., 2016, 138, 173-185.

88 V. Grande, B. Soberats, S. Herbst, V. Stepanenko and F. Wuerthner, Chem. Sci., 2018, 9, 6904-6911.

89 K. Sato, W. Ji, L. C. Palmer, B. Weber, M. Barz and S. I. Stupp, J. Am. Chem. Soc., 2017, 139, 8995-9000.

90 G. Wang, B. Tang, Y. Liu, Q. Gao, Z. Wang and X. Zhang, Chem. Sci., 2016, 7, 1151-1155.
91 J. Chen, T. Wang and M. Liu, Chem. Commun., 2016, 52, 6123-6126.

92 L. Ji, G. Ouyang and M. Liu, Langmuir, 2017, 33, 12419-12426.

93 H. Jiang, L. Zhang, J. Chen and M. Liu, ACS Nano, 2017, 11, 12453-12460.

94 S. Wang, H. Jiang, L. Zhang, J. Jiang and M. Liu, ChemPlusChem, 2018, 83, 1038-1043.

95 X. Zhu, P. Duan, L. Zhang and M. Liu, Chem. - Eur. J., 2011, 17, 3429-3437.

96 K. Chen, T. Jiao, J. Li, D. Han, R. Wang, G. Tian and Q. Peng, Langmuir, 2019, 35, 3337-3345.

97 P. Xing, Y. Li, S. Xue, S. Z. F. Phua, C. Ding, H. Chen and Y. Zhao, J. Am. Chem. Soc., 2019, 141, 9946-9954.

98 A. Maity, M. Gangopadhyay, A. Basu, S. Aute, S. S. Babu and A. Das, J. Am. Chem. Soc., 2016, 138, 11113-11116.

99 Y. Sun, S. Li, Z. Zhou, M. L. Saha, S. Datta, M. Zhang, X. Yan, D. Tian, H. Wang, L. Wang, X. Li, M. Liu, H. Li and P. J. Stang, J. Am. Chem. Soc., 2018, 140, 3257-3263.

100 S. Shin, S. Lim, Y. Kim, T. Kim, T.-L. Choi and M. Lee, J. Am. Chem. Soc., 2013, 135, 2156-2159.

101 R. Oda, I. Huc, M. Schmutz, S. J. Candau and F. C. MacKintosh, Nature, 1999, 399, 566-569.

102 D. Liu, G. Zhang, B. Gao, B. Li and L. Wu, Dalton Trans., 2019, 48, 11623.

103 P. Saokham and T. Loftsson, Int. J. Pharm., 2017, 516, 278-292.

104 G. Chen and M. Jiang, Chem. Soc. Rev., 2011, 40, 2254-2266.

105 K. A. Connors, Chem. Rev., 1997, 97, 1325-1357.

106 X. Wang, M. Li, P. Song, X. Lv, Z. Liu, J. Huang and Y. Yan, Chem. - Eur. J., 2018, 24, 13734-13739.

107 S. Yang, Y. Yan, J. Huang, A. V. Petukhov, L. M. J. KroonBatenburg, M. Drechsler, C. Zhou, M. Tu, S. Granick and L. Jiang, Nat. Commun., 2017, 8, 15856.

108 F. Xie, G. Ouyang, L. Qin and M. Liu, Chem. - Eur. J., 2016, 22, 18208-18214.

109 Y. Zhang, D. Yang, J. Han, J. Zhou, Q. Jin, M. Liu and P. Duan, Langmuir, 2018, 34, 5821-5830.

110 L. Ji, Y. Sang, G. Ouyang, D. Yang, P. Duan, Y. Jiang and M. Liu, Angew. Chem., Int. Ed., 2019, 58, 844-848.

111 L. Ji, Q. He, D. Niu, J. Tan, G. Ouyang and M. Liu, Chem. Commun., 2019, 55, 11747-11750.

112 B. Zhang, L. Yue, Y. Wang, Y. Yang and L. Wu, Chem. Commun., 2014, 50, 10823-10826.

113 R. Sun, C. Xue, X. Ma, M. Gao, H. Tian and Q. Li, J. Am. Chem. Soc., 2013, 135, 5990-5993.

114 P. Duan, L. Qin and M. Liu, Langmuir, 2011, 27, 1326-1331.

115 L. Qin, P.-F. Duan and M.-H. Liu, Chin. Chem. Lett., 2014, 25, 487-490. 